\title{
Near-infrared Spectroscopy of Brown Dwarf and Planetary-Mass Members in Upper Scorpius
}

\author{
Nicolas Lodieu ${ }^{1,2}$ \\ ${ }^{1}$ Instituto de Astrofísica de Canarias (IAC), Calle Vía Láctea s/n, E-38200 La Laguna, \\ Tenerife, Spain \\ email: nlodieu@iac.es \\ ${ }^{2}$ Departamento de Astrofísica, Universidad de La Laguna (ULL), E-38206 La Laguna, \\ Tenerife, Spain
}

\begin{abstract}
In these proceedings, I present new VLT/X-shooter near-infrared spectroscopy of brown dwarf and planetary-mass candidates with masses below 30 Jupiter masses identified in a deep VISTA $Z Y J$ survey of 13.5 square degrees in the Upper Scorpius (USco) association. These spectra represent new benchmarks at 5-10 Myr to compare with known and future discoveries of members in nearby moving groups and other young regions.
\end{abstract}

Keywords. Brown dwarfs — planetary-mass objects — surveys — photometry — spectroscopy

\section{The Upper Scorpius association}

USco is part of the nearest OB association to the Sun, Scorpius Centaurus, located at $145 \mathrm{pc}$ from the Sun (de Bruijne et al. 1997). The combination of its close distance, young age (5-10 Myr; Preibisch \& Zinnecker 1999; Pecaut et al. 2012; Song et al. 2012), and mean proper motion distinct from field stars makes it an ideal ground to search for cool brown dwarfs. Various groups have examined the association in X-rays and at optical and infrared wavelengths to identify bona-fide members. Spectroscopy of hundreds of stars and substellar members is now available, allowing us to characterise in-depth their binary and disk properties. The mass function of the association is very similar to the field mass function, from high-mass stars all the way to the substellar regime (Preibisch \& Zinnecker 2002; Lodieu et al. 2011).

\section{Photometric selection of brown dwarf member candidates}

We conducted a deep $Z Y J$ survey of 13.5 square degrees of USco with VISTA (Visible and Infrared Survey Telescope for Astronomy) in April/May 2012 in service mode under good conditions (Lodieu et al. 2013b). Our VISTA survey is $100 \%$ complete down to 22.0, 21.2 , and $20.5 \mathrm{mag}$ in $Z, Y$, and $J$, respectively. We complemented this dataset with additional $H$ and $K$ photometry as well as proper motions from the UKIDSS Galactic Clusters Survey (Lawrence et al. 2007). We identified 67 bona-fide $Z Y J$ candidates in multiple colour-magnitude diagrams (Fig. 1) as well as five $Y J$-only candidates with $J$ band magnitudes in the 14-20 mag range, corresponding to masses between 30 and 5 Jupiter masses according to state-of-the-art atmospheric models. We concluded that the USco mass function in the planetary-mass regime might be flat or decreasing by counting the number of potential members in the 20-10 and 10-5. Jupiter mass bins. 



Figure 1. Colour-magnitude diagrams used to identify brown dwarf members of the USco association combining photometry from our deep VISTA $Z Y J$ survey and public $H K$ photometry from the UKIDSS Galactic Clusters Survey (Figure from Lodieu et al. 2013b).

\section{VLT/X-shooter near-infrared spectroscopy}

We obtained near-infrared (1.0-2.4 microns) intermediate-resolution ( $\mathrm{R} \sim 3900)$ spectroscopy with the X-shooter instrument on the Very Large Telescope (VLT) on 10-14 April 2015 in visitor mode. We observed 15 USco candidate members with $J=17-19.5$ mag, equivalent to masses below the deuterium-burning limit. Moreover, half of the candidates with $J=15-17 \mathrm{mag}$ (i.e. masses in the $\sim 20-10$ Jupiter mass range) identified in our deep VISTA survey are already confirmed members with optical spectral types (Luhman \& Mamajek 2012). Those candidates have also VLT/X-shooter spectra available from the European Southern Observatory archive from an independent programme. All spectra show weak alkali lines and peaked $H$-band, signs of youth (Fig. 2). We also observe a diversity among the near-infrared spectra of these new members. We estimate preliminary spectral types in the L3-L7 range by comparison with known young members of nearby association and clusters (e.g. Lafrenière et al. 2010; Zapatero et al. 2014). 




Figure 2. VLT/X-shooter near-infrared of 15 USco member candidates confirmed spectroscopically.

We compared our spectra to intermediate-age and very low gravity members of moving groups, suggesting that the latter might have dusty atmospheres or harbour discs. These results will be presented in more details in a future paper (Lodieu et al. 2015, in prep).

\section{References}

de Bruijne et al. 1997, ESA SP-402: Hipparcos - Venice '97, p 575

Lafrenière, Jayawardhana, \& van Kerkwijk 2010, ApJ, 719, 497

Lawrence, A., Warren, S. J., Almaini, O., et al. 2007, MNRAS, 379, 1599

Lodieu, N., Dobbie, P. D., \& Hambly, N. C. 2011, A\&\&A, 527, 24

Lodieu, N., Dobbie, P. D., Cross, N. J. G., et al. 2013b, MNRAS, 435, 2474

Lodieu, N. 2013a, MNRAS, 431, 3222

Luhman, K. L. \& Mamajek, E. E. 2012, ApJ, 758, 31

Pecaut, M. J., Mamajek, E. E., \& Bubar, E. J. 2012, ApJ, 746, 154

Preibisch, T. \& Zinnecker, H. 1999, AJ, 117, 2381

Preibisch, T. \& Zinnecker, H. 2002, AJ, 123, 1613

Song, I., Zuckerman, B., \& Bessell, M. S. 2012, AJ, 144, 8

Zapatero Osorio et al. 2014, A\&SA, 568, 77

\section{Acknowledgements}

My current contract is funded by the Ramón y Cajal fellowship number 08-303-01-02. I also acknowledge funding from an IAC internal project and another project financed by the Spanish Ministry of Economy and Competitiveness (MINECO).

\section{Discussion}

L. MALO: You should measure radial velocities before claiming that these sources are members of the USco association 
AUThor: These candidates are selected in the central region of USco. We selected them photometrically using 5-band photometry $(Z Y J H K)$ and proper motions. The sequence is well-separated from the field stars in this part of the association not affected by reddening. The weak alkali lines and peaked $H$-band confirm their youth. We are pretty confident that they are bona-fide members of USco. Nonetheless we will attempt to mesure radial velocities from our X-shooter spectra comparing with the latest models available to us. This will be presented in a future paper (Lodieu et al. 2015, in prep).

M. LiU: Do you observe any difference in the sequence of members that might be due to age spread in the USco association?

Author: We did not look into this. The VISTA survey covers only 13.5 square degrees in the central part of the association so we do not expect much spread. However, this is a good idea to check using our latest list of member candidates over the full association (Lodieu 2013a) once the map generated by Pecaut \& Mamajek is published. 